\title{
A high-throughput Raman notch filter set
}

G. J. Puppels, A. Huizirga, H. W. Krabbe,H. A. de Boer, G. Gijsbers, and F. F. M. de Mul

(Received $12 \mathrm{July} 1990$; accepted for publication 10 August 1990)

A chevron-type Raman notch fiter (RNF) set is described. 1t combines a high signal throughput (up to $90 \%$ around $1600 \mathrm{~cm}^{-1}$ and $>80 \%$ between and 700 and $2700 \mathrm{~cm}^{-1}$ ) with a laser line suppression of $10^{8}-10^{9}$. The filter set can be used to replace the first two dispersion stages in triple-stage Raman monochromators commonly employed in multichannel detection systems. This yields a gain in intensity of the detected Raman signal of a factor of 4 . It is shown that in Raman spectrometers with a backscatter geometry, the filter set can also be used to optically couple the microscope and the spectrometer. This leads to a further increase in signal intensity of a factor of $3-4$ as compared to the situation where a beam splitter is used. Additional advantages of the RNF set are the fact that signal throughput is almost polarization independent over a large spectral interval and that it offers the possibility to simultaneously record Stokes and anti-Stokes spectra.

\section{INTRODUCTION}

In Raman spectroscopic experiments Rayleigh scattering and reflections may cause the intensity of the laser light, collected by the collection optics, to be many orders of magnitude higher than the intensity of the collected Raman scattered light. Suppression of this laser light while maintaining a high throughput of Raman signal in the wave-number region of interest is therefore an important issue in the design of Raman instrumentation. Most Raman spectrometers are equipped with a double- or triplewavelength dispersion stage monochromator (TDSM). The main advantages are that such a spectrometer can be used in a wide excitation wavelength region, and allows measurements at very low wave numbers. Disadvantages are the relatively low signal throughput, the strong polarzation dependence of the throughput (especially when holographic gratings are used), and the high price. In a TDSM the first two stages serve to select the desired part of the spectrum and suppress the laser line. In practice these have a combined throughput of only about $20 \%-$ $25 \%$ for Raman scattered light. Fiters can in most cases provide a good alternative for laser line suppression. Various possibilities have been described. Above $\sim 1200 \mathrm{~cm}^{-1}$ a set of well-chosen cutoff color-glass filters often is the easiest solution. ${ }^{1,2}$ A promising, but from a technological viewpoint complicated, device is the crystailine colloidal Bragg diffraction filter. ${ }^{3}$ In Ref. 4 it is described how a window in the absorbtion spectrum of a solution of quinoline in methanoi was used. The solution absorbed the laser light of $231.5 \mathrm{~nm}$ but had a transmission window of $40 \%-50 \%$ for the Raman signal between 900 and 1800 $\mathrm{cm}^{-1}$.

As part of a program concerning the development of a very sensitive confocal Raman microscope (CRM), a chevron-type Raman notch filter (RNF) set was developed, employing dielectric bandpass filters. It yields a laser line suppression of $-10^{8}-10^{9}$ and a throughput of Raman signal of up to $90 \%$. So far we have manufactured filter sets for the suppression of laser light of 514.5 and $660 \mathrm{~nm}$ but the design can be used to construct a filter set for any desired wavelength.

\section{DESIGN}

The RNF set consists of two identical dielectric bandpass filters, mounted parallel at an angle $\alpha$ with respect to the incoming parallel light beam [Figs. 1 (A) and 1(B)]. The light is repeatedly refected between the filters and the pass-band characteristics at angle $\alpha$ are such that at each refection there is optimum transmission of laser light, similar as in the device described in Ref. 6 . The laser light is in that way separated from the Raman scattered light, which is very effectively refiected. After passing the fliter set a first time an antirefiection-coated $90^{\circ}$ prism reverses the direction of the light so that it passes through the filter set a second time. The light beam then emerges from the filter set at the same height and parallel to the incoming light beam, independent of the tilting angle $\alpha$ of the filter set. This feature allows easy incorporation into any Raman setup and easy tuning (of angle $\alpha$ ) of the filter set for maximum suppression of laser light. The RNF set can be designed to accommodate parallel light beams of any diameter, although of course the technical problems encountered in the production of the filters increase with the dimensions of the filters. The length $L$ of the bandpass filters and the distance $d$ between them needed to obtain the desired number of reffections $N_{r}$ for a beam of diameter $D$ at a tilting angle $\alpha$ can be obtained from the equations given below [see Fig. 1(B)]

$$
\begin{aligned}
& l=W N_{r} /(4 \cos \alpha), \\
& d=W /(2 \sin \alpha)
\end{aligned}
$$

(where $W>D$ is demanded).

\section{MATERIALS AND METHODS}

The bandpass filters used were designed and produced in the thin-film laboratory of our department. Seventeen 


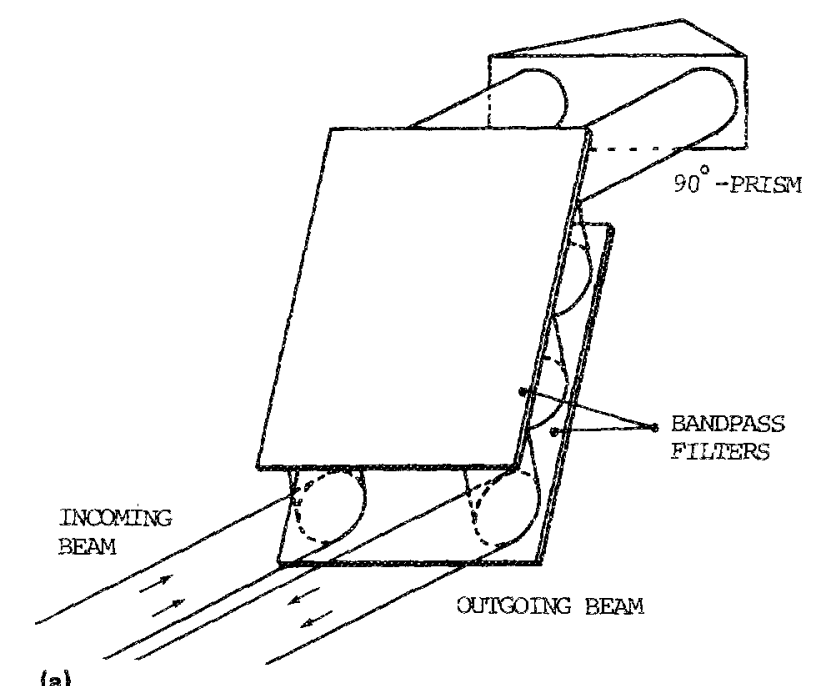

(a)

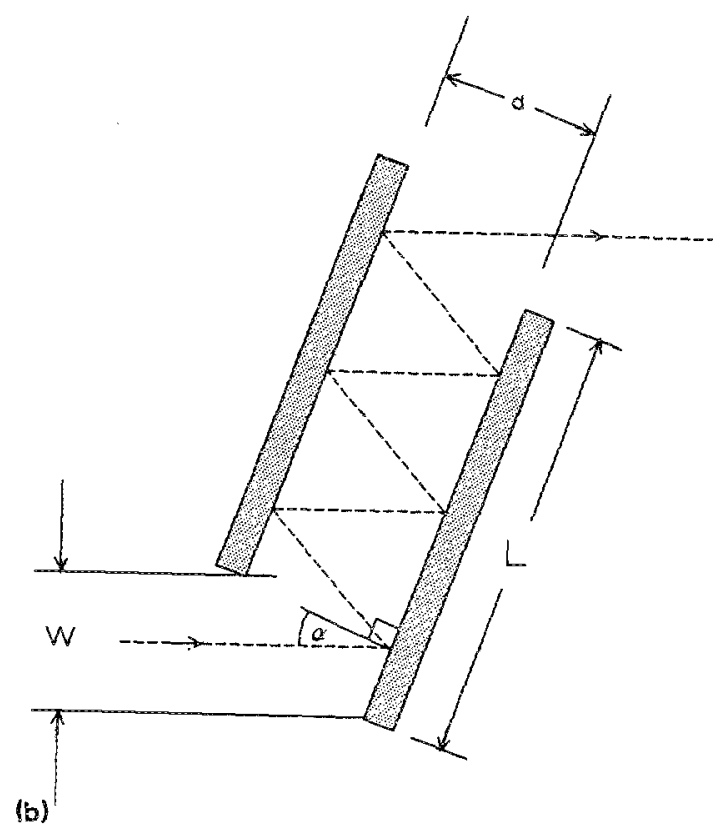

FIG. 1. (A) The chevron-type Raman notch filter set. (B) Side view.

hard nonabsorbing dielectric layers were used (layers $1,3,5,7, \ldots, 17: \lambda / 4 \mathrm{ZnS} ; 2,4,6,8,12,14$, and $16: \lambda / 4 \mathrm{ThF}_{4} ; 10$ : $\lambda / 2 \mathrm{ThF}_{4}, \lambda=\lambda_{\text {passband }}$ corrected for tilting angle $\alpha$ ) on glass substrates $(50 \times 50 \times 6 \mathrm{~mm})$.

Transmission curves of the filters were recorded on a Beckman DU-8 spectrophotometer (not shown). The values were corrected for reflection losses at the rear surface of the filter substrates. At an angle of $17^{\circ}$ transmittance for the $514.5-\mathrm{nm}$ argon-ion laser line is $76 \%$ for $p$-polarized light and $71 \%$ for $s$-polarized light. $p$ and $s$ polarization refer to the polarization with respect to the plane through incoming and reflected beams in the RNF set. Full width at half maximum (FWHM) is $\sim 5 \mathrm{~nm}$. For a large part of the Raman spectrum $\left(700-2700 \mathrm{~cm}^{-1}\right)$ reflection is above $99 \%$, peaking around $1600 \mathrm{~cm}^{-1}$ at $99.8 \%$.

The suppression of laser light by the RNF set was determined by comparing with a set of calibrated absorbtion neutral density filters (optical density of each filter

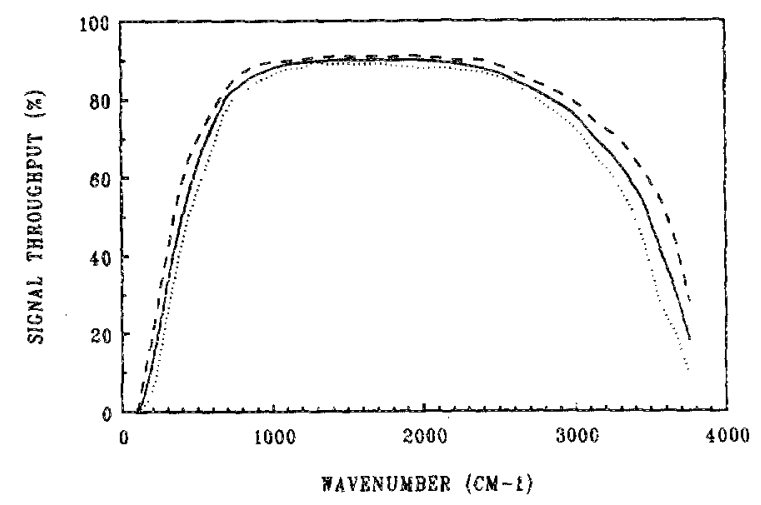

FIG. 2. Signal throughput of the RNF set (--mpolarized light;...ppolarized light;---s-polarized light).

between 1 and 2 ). The neutral density filters were calibrated using a Scientech 361 power meter at $514.5 \mathrm{~nm}$.

For these measurements the RNF set was incorporated in a Raman spectrometer schematically depicted in Fig. $2(\mathrm{~A})$. Laser light was focused on a mirror with the microscope objective. The reflected light was collected and (partiy) coupled into the spectrometer. The RNF set was then tuned for maximum suppression of this laser light. The intensity of the remaining laser light was measured with a liquid-nitrogen-cooled CCD camera (model $1 \mathrm{CCD}$ camera with an EEV P 8603 B chip, Wright Instruments Ltd.). Then the mirrors $M 1$ and $M 2$ were removed so that the laser light did not pass through the filter set, and replaced by neutral density filters. From the laser light intensity remaining and the extinction values of the neutral density filters the suppression of laser light by the RNF-set could be determined. This was done for $p$ and $s$-polarized laser light.

Signal throughput of the filter set was determined in a similar way. For this the in-base illumination system of the microscope frame (Nikon Optiphot) was used. The continuous spectrum of the light collected by the microscope objective was recorded with the RNF set in place and compared with the situation with mirrors $M 1$ and $M 2$ removed, so that the light is directly coupled into the spectrometer. An argon spectral calibration lamp (Oriel model 6030) was used for wave-number calibration of the spectra. In order to determine the dependence of the signal throughput on the polarization of the light, a polarizer (Spindler \& Hoyer) was installed in front of mirror $M 1$, and the measurements described above repeated for $p$ - and $s$-polarized light. The error in the transmission measurements was determined from the spread in the values obtained in three different measurements. For each of the measurements the RNF set was detuned and then tuned again for optimum laser line suppression.

The simultaneous recording of the Stokes and antiStokes Raman spectrum shown in Fig. 3 was recorded on a Raman microspectrometer constructed in our laboratory. The optical scheme of this instrument is similar to the one shown in Fig. 2(B). It is equipped with a photodiode array (EG\&G Reticon RL512SF) with an image intensifier (DEP XX1450) for signal detection. 


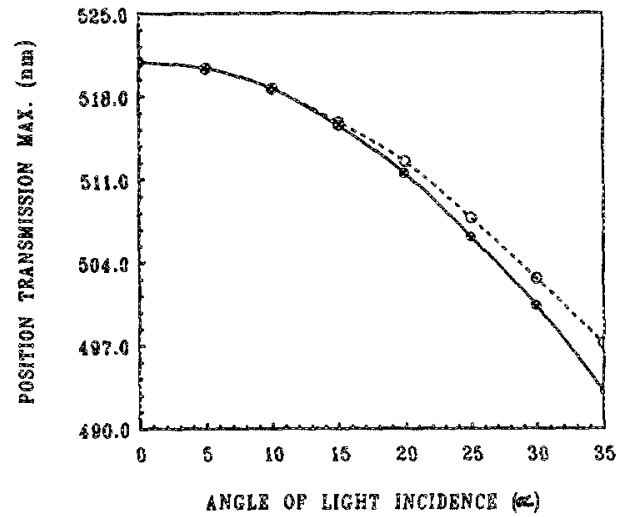

FIG. 3. Position of the passband of the dielectric bandpass filters for $p$ and $s$ polarization as a function of tilting angle $\alpha(O$ p-polarized light; s-polarized light).

\section{PERFORMANCE}

The performance of a RNF set like this depends entirely upon the characteristics of the bandpass filters. Throughput $T_{\lambda}$ at wavelength $\lambda$ is obtained from

$$
T_{\lambda}=c_{l} R_{\lambda}^{N_{r}} \text {, }
$$

where $R_{\lambda}$ is the reflection of the bandpass filter at wavelength $\lambda, N$, is the number of reflections used in the notch filter, and $c_{l}$ is the factor representing losses at the prism and mirrors $M 1$ and $M 2$.

Figure 4 shows the throughput for Raman light recorded for a RNF set employing the bandpass filters described above, using 16 reflections. Maximum transmission is $90 \% \pm 3 \%$. s-polarized light is reflected slightly more efficient than $p$-polarized light. After 16 reflections a difference in throughput for the two orthogonal polarizations becomes noticeable especially in the wings of the throughput curves. However, in a wide spectral region ( $600-2600$ $\mathrm{cm}^{-1}$ ) this difference is less than $10 \%$, which compares very favorably with the polarization dependence of (double) grating dispersion stages. ${ }^{7}$

Using 16 reflections we have achieved a laser line suppression of $10^{8}-10^{9}\left(\sim 10^{9}\right.$ for $p$-polarized and $\sim 10^{8}$ for $s$-polarized laser light). Of course a RNF set like this can be optimally employed for only one laser line. However, performance is not significantly affected in a tuning range of $5 \mathrm{~nm}$ (by adjusting $\alpha$ ), so that the specifications for the production of the filters are not extremely critical with respect to the position of the passband.

The filters can easily be replaced when another laser source is used for excitation. Mechanical and temperature (in the range $15-25^{\circ} \mathrm{C}$ ) stability are such that no readjustments of the RNF set are needed as long as the position and angle of the incoming light beam are unchanged.

An important aspect of this type of bandpass filter is that at angles $\alpha>0$ the passband position tends to diverge for $p$ and $s$ polarized light (Fig. 5). Also peak transmission of the passband decreases and bandwidth increases for $\alpha$ $>0$. All of these effects negatively affect the RNF characteristics. Therefore, $\alpha$ should be kept as small as possible.
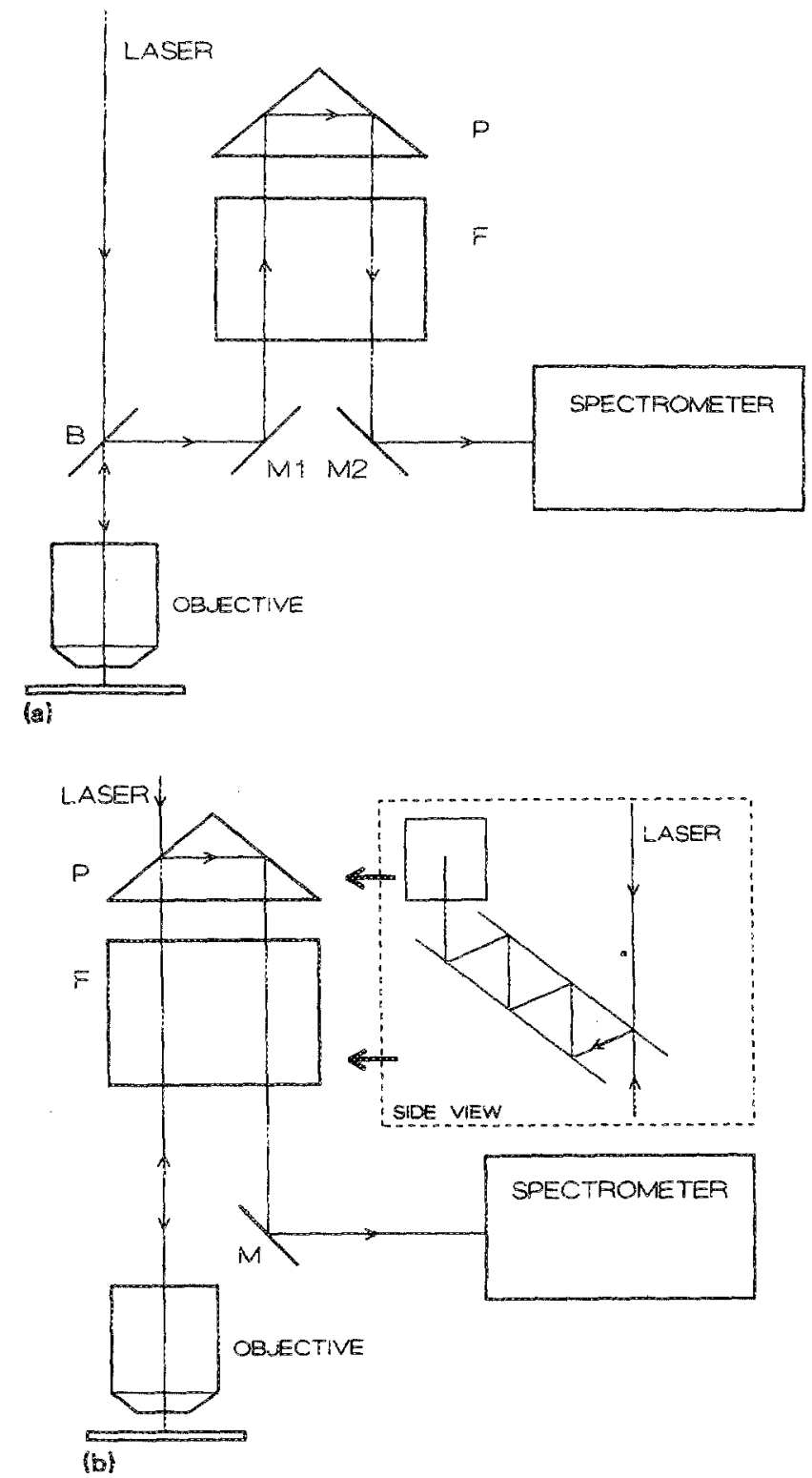

FIG. 4. Two setup configurations empioying the RNF set. Abbreviations: $F$ : RNF-set, $B$ : beamsplitter, $M:$ mirror, $P$ : prism. (A) RNF set replaces another filter stage. (B) RNF set replaces another fiter stage and couples microscope and spectrometer.

An angle $\alpha$ between $15^{\circ}$ and $20^{\circ}$ was found to be the best compromise between optimum filter performance on one hand and technical feasibility and ease of operation on the other. It is expected that with proper facilities for maintaining precise control of the filter production process it will be possible to develop bandpass filters which allow recording of Raman spectra in the lower wave-number region down to $100 \mathrm{~cm}^{-1}$.

\section{APPLICATION}

The RNF set can be incorporated in a Raman setup in different ways. It can replace another laser line suppression stage. But in setups with a backscatter geometry (such as in a Ramar microscope) it can also replace a beamsplitter, otherwise needed for the incoupling of laser light to the 


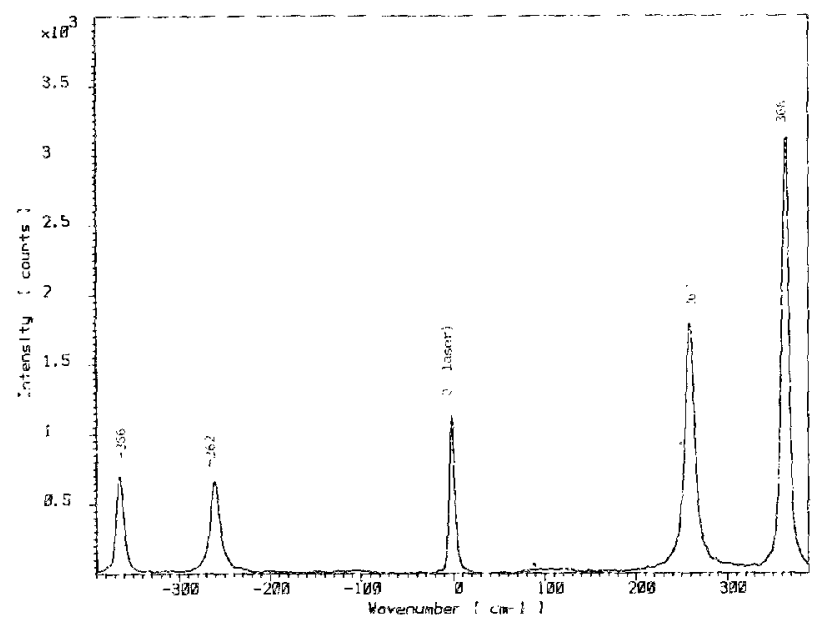

FIG. 5. Simultaneously recorded Stokes and anti-Stokes Raman spectra of $\mathrm{CHCl}_{3}$ (not corrected for the wave-number-dependent signal throughput of the spectrometer).

sample and the outcoupling of collected Raman light to the spectrometer [Figs. 2(A) and 2(B)]. With the same laser output power a further gain in signal intensity of a factor of $3-4$ (depending on fitter trarsmission at the passband) can be achieved in this manner.

Using filters with good reflection at both sides of the passband it is possible to record Stokes and anti-Stokes spectra simultaneousiy, as shown in Fig. 3 , for $\mathrm{CHCl}_{3}$.

\section{DISCUSSION}

We have shown that it is possible to achieve a signifcant increase in throughput of Raman light using the RNF set presented here. Comparison with the widely used filter stage of a TDSM shows a gain in throughput of a factor of 4. Employment of the RNF set as in Fig. $4(B)$ increases signal intensity more than ten-fold. This enables the recording of higher-quality Raman spectra or the use of a lower laser intensity on samples. The possibility to record ant-Stokes and Stokes Raman spectra simultaneousiy and the polarization-independent throughput are valuable extra advantages.

'A. Deffontaine, M. Bridoux, M. Delhaye, E. Da Silva, and W. Hug, Rev. Phys. Appl. 19, 415 (1984).

${ }^{2}$ M. P. F. Bristow, Appl. Opt. 18, 952 (1979).

${ }^{3}$ P. L. Flangh, S. E. O'Donnell, and S. A. Asher, Appl. Spectrosc. 38, 847 (1984).

${ }^{4}$ M. Baek, W. H. Nelson, D. Britt, and J. F. Sperry, Appl. Spectrosc. 42, $1312(1988)$.

${ }^{5}$ G. J. Puppels, F. F. M. de Mul, C. Otto, J. Greve, M. Robert-Nicoud, D. J. Arndt-Jovin, and T. Jovin, Nature 347, 301 (1990).

${ }^{6}$ K. Togichi, Y. Hirano, Y. Hiratsuka, and T. Edamura, Proceedings of the IXth International Conference on Raman Spectroscopy (Chemical Socicty of Japan, Tokyo, Japan, 1984).

${ }^{7}$ See, e.g., Diffraction gratings, ruled and hologrophic Ilandbook (Jobin Yvon S.A., Longumeau, France). 